\title{
Stark broadening measurements in plasmas produced by laser ablation of hydrogen containing compounds
}

\author{
Miloš Burger ${ }^{\mathrm{a}, *}$, Jörg Hermann ${ }^{\mathrm{b}}$ \\ ${ }^{a}$ University of Belgrade, Faculty of Physics, POB 44, 11000 Belgrade, Serbia \\ ${ }^{b}$ LP3, CNRS - Aix-Marseille University, 13008 Marseille, France
}

\begin{abstract}
We present a method for the measurement of Stark broadening parameters of atomic and ionic spectral lines based on laser ablation of hydrogen containing compounds. Therefore, plume emission spectra, recorded with an echelle spectrometer coupled to a gated detector, were compared to the spectral radiance of a plasma in local thermal equilibrium. Producing material ablation with ultraviolet nanosecond laser pulses in argon at near atmospheric pressure, the recordings take advantage of the spatially uniform distributions of electron density and temperature within the ablated vapor. By changing the delay between laser pulse and detector gate, the electron density could be varied by more than two orders of magnitude while the temperature was altered in the range from 6,000 to $14,000 \mathrm{~K}$. The Stark broadening parameters of transitions were derived from their simultaneous observation with the hydrogen Balmer alpha line. In addition, assuming a linear increase of Stark widths and shifts with electron density for non-hydrogenic lines, our measurements indicate a change of the Stark broadening-dependence of $\mathrm{H}_{\alpha}$ over the considered electron density range. The presented results obtained for hydrated calcium sulfate $\left(\mathrm{CaSO}_{4} \cdot 2 \mathrm{H}_{2} \mathrm{O}\right)$ can be extended to any kind of hydrogen containing compounds.
\end{abstract}

Keywords: Stark broadening; Spectra simulation; Hydrogen; Calcium; LIBS.

\section{Introduction}

Stark broadening of spectral lines is under investigation since the discovery of the effect in 1913. With the diversification of the available plasma sources and the increasing interest for plasma diagnostic tools, the theoretical and experimental studies dedicated to Stark broadening became popular in the 1960's [1,2]. Since that time, several review papers have been published to summarize the results obtained by a large number of research groups all over the world [3-7]. Despite of the numerous efforts in the past decades, precise Stark broadening parameters are still only partially available, even for the most prominent transitions. This is mainly due to the difficulties of calibrating the Stark broadening measurements using an alternative and independent measurement method. Recently, Thomson scattering was applied to measure electron density and temperature in laser-produced plasmas [8,9]. However, the application of this method to high-density thermal plasmas

${ }^{*}$ Corresponding author: milosb@ ff.bg.ac.rs is doubtful due to electron heating by the probe laser radiation. From the theoretical point of view, there does not exist any model that enables accurate calculations of Stark broadening over a large electron density range, as illustrated by Griem for $\mathrm{H}_{\alpha}$ [10].

The lack of accurate Stark broadening data and the need of further developments in appropriate models motivate the related research in different types of plasmas. With respect to arcs, sparks or other electrical discharges, the plasmas produced by pulsed lasers are historically younger. This is mainly due to the technological development of laser sources: reliable pulsed lasers that generate highly reproducible plasmas are available since the last two decades only. In addition, the small size and the fast expansion dynamics present particular difficulties for plasma diagnostics. With the invention of gated detectors and the development of applications such as laser-induced breakdown spectroscopy (LIBS), the investigation of plasmas produced by pulsed laser ablation stimulated a strongly growing interest in the past years. The small size and the large initial density now appear as advantages, since the former property limits the optical thickness of plasma emission, and the latter 
feature favors the establishment of local thermal equi- 95 librium $[11,12]$.

The expansion dynamics of plasmas produced by laser ablation strongly depend on the irradiation conditions and the surrounding atmosphere. The use of infrared radiation favors the absorption of laser photons by the background gas, leading to an elongated shape of the plasma [13]. This condition enables rapid intermixing of the ablated vapor with the surrounding atmosphere [14]. Contrarily, the use of shorter wavelength radiation increases the laser-material energy coupling. The plasma screening effect $[15,16]$ is reduced, and the plasma is characterized by a hemispherical shape [17]. If, in addition to the use of the short laser wavelength, the ablation process occurs in an argon atmosphere, the ablation plume appears spatially almost uniform. This was illustrated by the analysis of the spectral shapes of resonance lines and strongly Stark-shifted transitions [18].

Stark broadening parameters of calcium lines are of interest to laboratory plasma diagnostics, as well as for theoretical modeling. In LIBS plasmas for example, $\mathrm{Ca}$ is often present as an impurity. Also, due to its large abundance all over the universe, calcium presents a constituent of many stellar plasmas, and $\mathrm{Ca}$ and $\mathrm{Ca}^{+}$ lines are of a great importance in astrophysics [19]. The most intense lines and in particular the ionic resonance lines were investigated extensively in the past [20-31]. The resonance lines are generally strongly self-absorbed, and their practical usage for plasma diagnostics is often doubtful. Stark broadening calculations, based on the semiclassical perturbation formalism, have been performed for many $\mathrm{Ca}[19,32]$ and $\mathrm{Ca}^{+}$transitions $[33,34]$. The correlation of Stark broadening with the energy gap between the upper-level of the transition and the ionization potential was also investigated [35]. However, Stark parameters of many Ca transitions in the visible and UV ranges are still missing in literature.

In the present work, we take advantage of the spatially uniform character of the plasma produced by UV nanosecond laser ablation in argon at near atmospheric pressure. Samples of hydrated calcium sulfate were ablated to obtain spectral line emission from hydrogen, calcium, oxygen and several impurities. Comparing ${ }^{133}$ the measured emission spectrum to the spectral radiance computed for a uniform plasma in local thermodynamic equilibrium, we were able to characterize the plasma and to deduce the Stark broadening parameters for many atomic and ionic lines. With respect to the traditional methods based on space-resolved spectroscopic measurements and complex data analysis via Abel in- ${ }_{138}$ version $[20,28,36]$, the presented method appears eas- 139 ier to handle and gives rapid access to a large number of data. Indeed, using an echelle spectrometer of large resolving power, the recording of a few spectra at different delays enables the determination of Stark broadening parameters of a large number of spectral lines.

\section{Method and calculation details}

\subsection{Principle of Stark broadening measurements}

The method for the measurement of Stark broadening parameters consists of the following three successive steps: $(i)$ the plasma temperature $T$, the electron density $n_{e}$, and the relative fractions of elements $C$ were deduced for spectra recorded at different times (delay between laser pulse and detector gate) using the iterative procedure decribed in Ref. [37]. Here, $n_{e}$ is deduced from $H_{\alpha}$ for which accurate electron density measurements are expected for $n_{e}$-values of the order of $10^{17} \mathrm{~cm}^{-3}$ [10]; (ii) Once the plasma is characterized, the Stark widths and shifts of non-hydrogenic lines are deduced from best agreement between measured and computed spectra. The plasma being characterized previously, the calculation of the line profiles accounts for Doppler- and resonance broadening; (iii) The Stark broadening parameters $w$ and $d$ of the non-hydrogenic lines were deduced from the linear increase of Stark width and shift with $n_{e}$.

\subsection{Calculation details}

Material ablation with pulsed lasers in a background gas at near atmospheric pressure leads to almost hemispherical expansion if the interaction of the laser beam with the gas is negligible, and the laser spot diameter is small compared to the plasma radius. In that case, the blast wave model may be applied to describe the plume expansion dynamics. The conditions are fulfilled for ultraviolet nanosecond laser pulses [13, 17]. If argon is used as a buffer gas, the spatial distributions of electron density and temperature within the ablated vapor are almost uniform and the spectral radiance of the plasma can be calculated using [37]

$$
I_{\lambda}=U_{\lambda}\left(1-e^{-\alpha L}\right),
$$

where $U_{\lambda}$ is the black-body spectral radiance, $L$ is the plasma diameter along the observation direction, and $\alpha$ is the absorption coefficient given by [1]

$$
\alpha(\lambda)=\pi r_{0} \lambda^{2} f_{l u} n_{l} P(\lambda)\left(1-e^{-h c / \lambda k T}\right) .
$$

Here, $r_{0}$ is the classical electron radius, $\lambda$ is the wavelength, $h$ is the Planck constant, $c$ is the vacuum light 


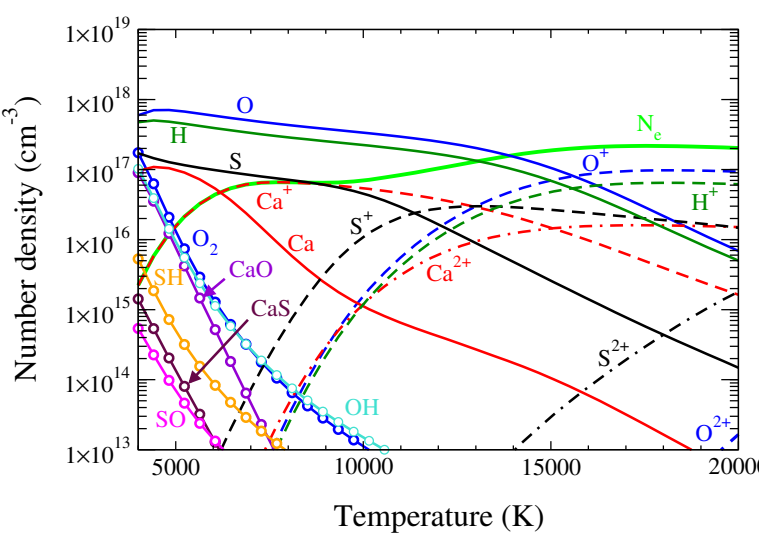

Figure 1: Number densities of species versus temperature computed for a $\mathrm{CaSO}_{4} \cdot 2 \mathrm{H}_{2} \mathrm{O}$ plasma in LTE at atmospheric pressure.

velocity, $k$ is the Boltzmann constant, $f_{l u}$ and $n_{l}$ are the absorption oscillator strength and the lower level population number density of the transition, respectively. The normalized line profile $P(\lambda)$ is calculated considering Doppler and Stark broadening that are the dominan mechanisms of spectral line broadening in strongly ionized laser-produced plasmas [38]. Depending on the relative values of Doppler and Stark widths, the line shapes are described by Gaussian, Lorentzian or Voigt profiles. The Doppler width is calculated according to plasma temperature and atomic mass of the emitting species The Stark width is obtained using [10, 39]

$$
\Delta \lambda_{\text {Stark }}=w\left(\frac{n_{e}}{n_{e}^{r e f}}\right)^{m},
$$

where $w$ is the Stark width at the reference electron density $n_{e}^{r e f}$. The Stark shift is obtained from Eq. (3) replacing $w$ by the Stark shift at the reference electron den- 205 sity $d$. We assumed linear dependence of Stark width 206 with electron density $(m=1)$ for all non-hydrogenic 207 lines. For the $\mathrm{H}_{\alpha}$ transition, different $m$-values were 208 reported in literature. The values obtained from the- 209 ory vary from 0.68 to 0.83 whereas $m \approx 0.35$ was re- 210 ported for experiments [10]. In the present work, we 211 use for $n_{e} \leq 1 \times 10^{17} \mathrm{~cm}^{-3}$ the expression proposed by 212 Gigosos et al. [40], using Eq. (3) with $w=1.10 \mathrm{~nm}, 213$ $n_{e}^{r e f}=1 \times 10^{17} \mathrm{~cm}^{-3}$, and $m=0.68$. For larger electron 214 densities, we use a slightly different expression with 215 a somewhat larger experimentally determined $m$-value 216 (see section 4.2). We stress that Stark broadening of $\mathrm{H}_{\alpha} \quad 217$ is recognized as a reliable tool for $n_{e}$-measurements in 218 laser-induced plasmas [41].

The lower level population number density in Eq. (2) is 220 obtained by calculating the plasma composition assum- 221 ing local thermodynamic equilibrium (LTE) [42]. The 222 number densities of plasma species computed for LTE are displayed in Fig. 1 for the elemental composition of the here investigated hydrated calcium sulfate sample. The calculations have been performed by setting the kinetic pressure of the plasma to atmospheric pressure [42]. As the pressure is kept constant, the atomic number densities of elements and thus the total atomic number density of the plasma decrease with increasing temperature.

In the considered temperature range, atomic and ionic species dominate the plasma composition. Molecular species significantly contribute to the plasma composition only for $T<5,000 \mathrm{~K}$. According to the moderate dissociation energies of the involved diatomic species [43], their number densities decrease rapidly with temperature, representing a fraction $<1 \%$ for $T=6,000 \mathrm{~K}$. For $T \leq 15,000 \mathrm{~K}$, neutral atoms are the dominating plasma species according to the large ionization potentials of the most abundant elements $\mathrm{H}$ and $\mathrm{O}$ [44]. The ionization potential of $\mathrm{Ca}$ being of only $6 \mathrm{eV}$, the electrons originate essentially from the ionization of calcium in the temperature range up to $9,000 \mathrm{~K}$. For $T>9,000 \mathrm{~K}$, the ionization of sulfur contributes significantly to the plasma ionization whereas $T>12,000 \mathrm{~K}$ is required to enable strong contributions of oxygen and hydrogen. The temperature dependence of the $\mathrm{Ca}^{2+}$ number density is similar to those of $\mathrm{O}^{+}$and $\mathrm{H}^{+}$. This is due to the ionization potential of $\mathrm{Ca}^{+}$that is close to the ionization energies of $\mathrm{O}$ and $\mathrm{H}$. For $T>15,000 \mathrm{~K}$, the ionic species dominate the plasma and $\mathrm{O}^{+}$and $\mathrm{H}^{+}$ions are the most abundant species.

\section{Experiment}

The experiments were carried out with a frequencyquadrupled Nd:YAG laser (Quantel, model Brilliant) delivering pulses of $4 \mathrm{~ns}$ duration and $40 \mathrm{~mJ}$ energy at the wavelength of $266 \mathrm{~nm}$. The laser pulse energy was attenuated to $6 \mathrm{~mJ}$ by turning the beam polarization with the aid of a half-wave plate and crossing through a polarization analyzer. The laser beam was focused onto the sample surface using a plano-convex lens of $150 \mathrm{~mm}$ focal length. According to a spot diameter of $100 \mu \mathrm{m}$ of the Gaussian beam, a laser fluence of about $80 \mathrm{~J} \mathrm{~cm}^{-2}$ was obtained on the sample surface. The pellet samples were prepared from commercially available hydrated calcium sulfate powder using a hydraulic press, and placed on a motorized sample holder in a vacuum chamber of $10^{-4} \mathrm{~Pa}$ residual pressure. During the experiments, the chamber was filled with argon at $5 \times 10^{4} \mathrm{~Pa}$ pressure. The plasma emission was captured by imaging the plume with two lenses of 150 and 35 


\subsection{Plasma characterization}

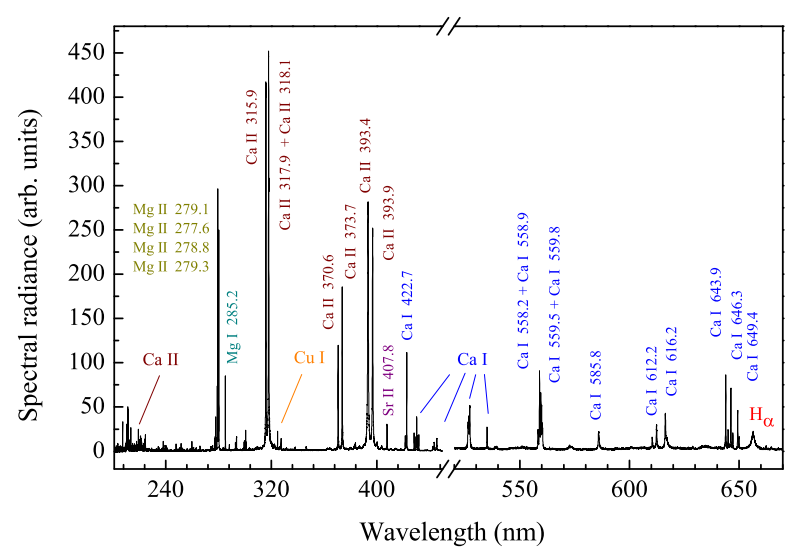

Figure 2: Spectrum recorded during ablation of hydrated calcium sulfate for $t=(475 \pm 75) \mathrm{ns}$

$\mathrm{mm}$ focal lengths onto the entrance of an optical fiber of $600 \mu \mathrm{m}$ diameter. The optical axis of the lenses was tilted by $15^{\circ}$ with respect to the surface normal. According to the image magnification of about 1:5, a cylindrical volume of about $3 \mathrm{~mm}$ diameter was observed. The fiber was coupled to the entrance of an echelle spectrometer (LTB, model Aryelle Butterfly) of $0.4 \mathrm{~m}$ focal length and a resolving power of $8.9 \times 10^{3}$. Photon detection was ensured using an intensified charge-coupled device matrix detector (Andor, model IStar). The spectral resolution of the apparatus was measured using a low-pressure argon-mercury lamp. An intensity calibration of the spectroscopic apparatus was performed in the visible and UV spectral ranges using a calibrated tungsten lamp (Oriel, model 63358) and a deuterium lamp (Heraeus, model DO544J), respectively.

The spectra were recorded for different delays of the detector gate $t_{g}$ with respect to the laser pulse. The gate width $\Delta t_{g}$ was adjusted for each delay so that $\Delta t_{g}<t_{g}$. We denote the measurement time $t=t_{g} \pm \Delta t_{g} / 2$. To enhance the signal-to-noise ratio, data acquisition was performed by averaging over 500 ablation events, applying 5 pulses to 100 different irradiation sites. The sites were separated by a distance of $150 \mu \mathrm{m}$.

\section{Results and discussion}

The emission spectrum of the plasma produced by laser ablation of hydrated calcium sulfate is displayed in Fig. 2 for the spectral ranges that exhibit the most significant investigated transitions. To facilitate the observation of the low-intensity transitions in the greenred range, the intensity scale was multiplied by a factor
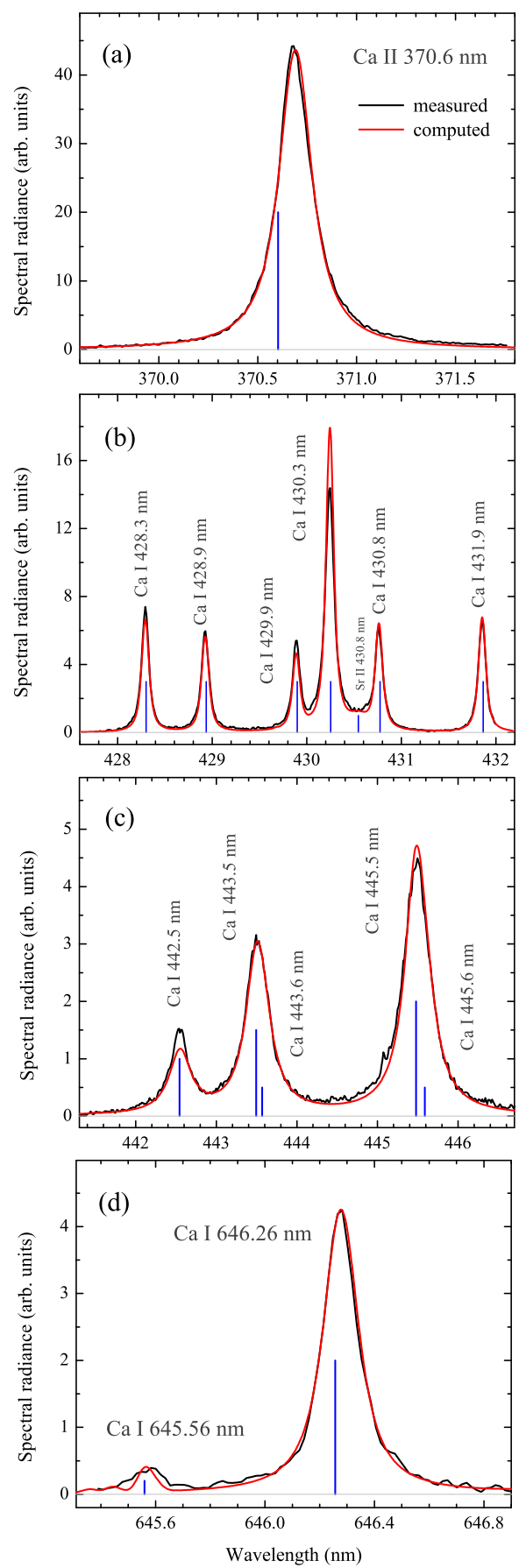

Figure 3: Measured spectrum (black line) and computed spectral radiance (red line) of various calcium transitions. The computed radiance was obtained for $T=12,200 \mathrm{~K}, n_{e}=1.6 \times 10^{17} \mathrm{~cm}^{-3}, L=0.65 \mathrm{~mm}$ and the elemental composition given in Table 1 . The blue lines denote the resonance wavelength of each transition. 
of 10 for that part of the spectrum. The plasma emission is dominated by spectral lines of singly charged calcium ions. In addition, transitions of $\mathrm{Ca}$ neutral atoms and of species from several impurity elements are observed. We identify the strongly broadened $\mathrm{H}_{\alpha}$ transition in the red range of the spectrum. The atomic fractions of both major and minor elements deduced from the best agreement between measured and computed spectra are given in Table 1.

The spectrum measured for $t=(475 \pm 75)$ ns (see Fig. 2) is displayed in Fig. 3 for several spectral ranges, together with the computed spectral radiance. We observe a good agreement between measured and computed spectral shapes for all presented transitions. It is shown that the lines are significantly broadened. Some transitions such as Ca II 370.6 nm (a) and Ca I 646.26 $\mathrm{nm}(\mathrm{d})$ are characterized by large Stark shifts. A slight difference in intensity is visible for some lines and in particular for Ca I $430.3 \mathrm{~nm}$. The mismatch is attributed to the low accuracy of the transition probabilities [44]. The time-evolution of the laser-induced plasma was investigated by recording spectra for different observation delays with respect to the laser pulse. The characteristic behavior is illustrated in Fig. 4 where the spectral shapes of $\mathrm{H}_{\alpha}$ (a), Ca I $585.74 \mathrm{~nm}$ (b), and $\mathrm{Mg}$ I $285.21 \mathrm{~nm}$ (c) are shown for different times. We observe strong broadening at early times followed by consecutive narrowing of the line profiles with increasing time. In addition, Ca I $585.74 \mathrm{~nm}$ and Mg I $285.21 \mathrm{~nm}$ exhibit large red-shifting at early times. Transitions of large Stark shift are characterized by asymmetric line shapes if they are emitted from a spatially non-uniform plasma [18]. Here, the symmetric shape observed for the Mg I $285.21 \mathrm{~nm}$ line (c) shows that the plasma is spatially uniform in agreement with

Table 1: Atomic fractions of the constituents of the hydrated calcium sulphate pellet deduced from the LIBS spectra $C_{L I B S}$. The reference values $C_{r e f}$ correspond to the chemical formula $\mathrm{CaSO}_{4} \cdot 2 \mathrm{H}_{2} \mathrm{O}$.

\begin{tabular}{ccc} 
Elmnt. & $C_{\text {LIBS }}(\%)$ & $C_{r e f}(\%)$ \\
\hline $\mathrm{Ca}$ & 9.1 & 8.3 \\
$\mathrm{~S}$ & 8.3 & 8.3 \\
$\mathrm{O}$ & 47 & 50.0 \\
$\mathrm{H}$ & 35 & 33.3 \\
$\mathrm{Cu}$ & 0.04 & - \\
$\mathrm{Fe}$ & 0.014 & - \\
$\mathrm{Li}$ & 0.017 & - \\
$\mathrm{Mg}$ & 0.4 & - \\
$\mathrm{Si}$ & 0.08 & - \\
$\mathrm{Sr}$ & 0.012 & -
\end{tabular}
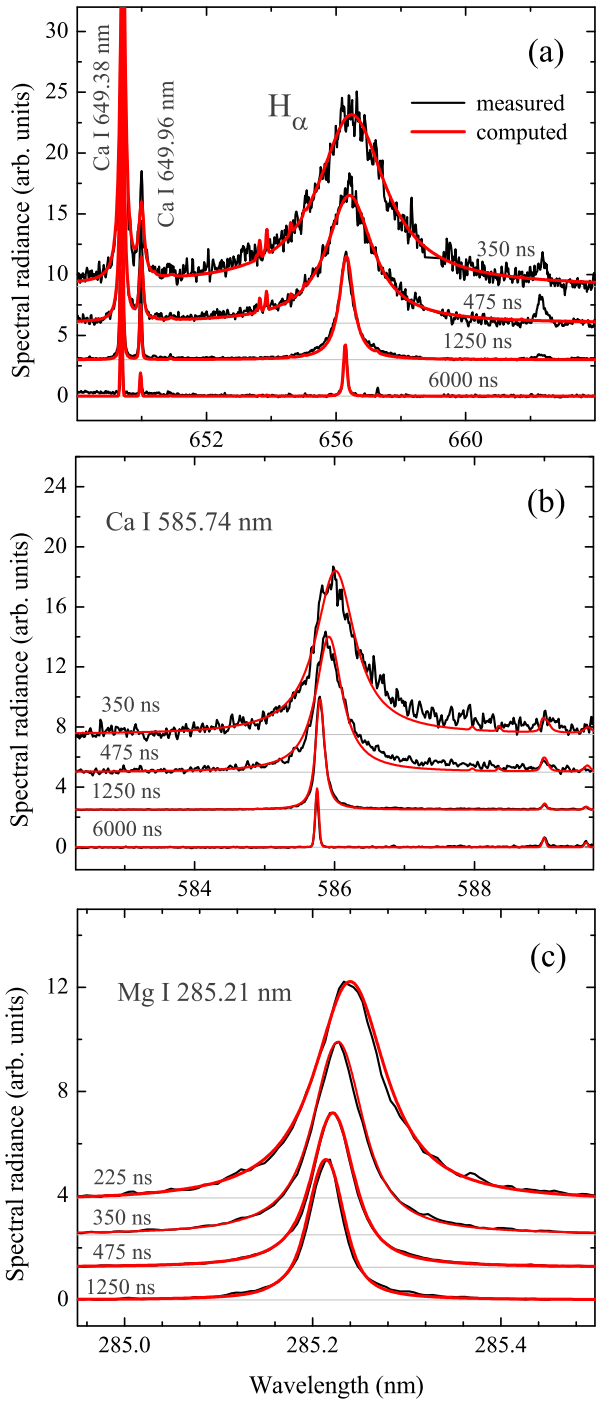

Figure 4: Measured (black line) and computed (red line) spectral radiance of several transitions for different observation times.

previous observations of LIBS plasmas produced in argon background gas [37, 45].

The plasma temperature evolution is illustrated by the Saha-Boltzmann plots displayed in Fig. 5. Here, $\epsilon$ is the emission coefficient deduced from the measurements using $\epsilon=\epsilon_{c} I_{m} / I_{c}$, where $\epsilon_{c}$ is the calculated emission coefficient, and $I_{m}$ and $I_{c}$ are the measured and computed line-integrated spectral radiances, respectively. As the computed radiance intrinsically accounts for self-absorption, the Saha-Boltzmann plot displayed in Fig. 5 is equivalent to the Boltzmann plot corrected for self-absorption presented by Bulajic et al. [46]. It is 


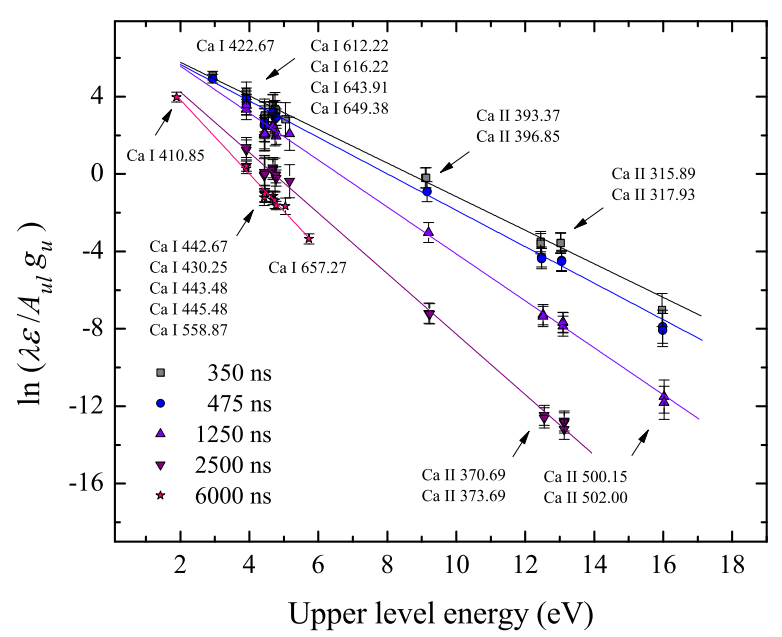

Figure 5: Saha-Boltzmann plots of calcium for various observation times.

shown that the population number densities of atomic and ionic excited species are well described by the equilibrium distribution for all measurement times.

The deduced values of temperature and electron density are shown in Fig. 6 as functions of time. The horizontal error bars represent the gate width, whereas the vertical error bars stand for the measurement uncertainties. According to Griem [10], electron density measurements using $\mathrm{H}_{\alpha}$ are most precise for $n_{e}$-values close to $10^{17} \mathrm{~cm}^{-3}$. The measurement error increases with distance from that value due to the uncertainty ${ }_{334}$ of the exponent $m$ (see Eq. 3). We estimated the $n_{e^{-}}{ }_{335}$ measurement error assuming uncertainties of $10 \%$ for ${ }_{336}$ the parameters $w$ and $m$ and of 5\% for the Stark width ${ }_{337}$ measurement. During the considered time-interval from 200 to $6000 \mathrm{~ns}$, the electron density decreases by ${ }_{339}$ more than two orders of magnitude from $5 \times 10^{17}$ to ${ }_{340}$ $3 \times 10^{15} \mathrm{~cm}^{-3}$, whereas the temperature diminishes from about 14,000 to $6,000 \mathrm{~K}$.

\subsection{Stark width and shift measurements}

The strong variation of electron density over the mea- ${ }_{34}$ sured time-interval and the spatially uniform character ${ }_{347}$ of the laser-produced plasma are now explored to mea- ${ }_{348}$ sure the Stark widths and shifts of spectral lines. We ${ }_{349}$ emphasize that the calculation of the spectral radiance 350 allows us to predict the optical thickness of each tran- 351 sition, and thus to exclude strongly self-absorbed lines 352 from the analysis. For some transitions such as reso- ${ }_{353}$ nance lines of neutral atoms, the optical thickness crit- ${ }_{354}$ ically depends on the observation delay. At early time, 355 when the plasma temperature is high and ionic species 356

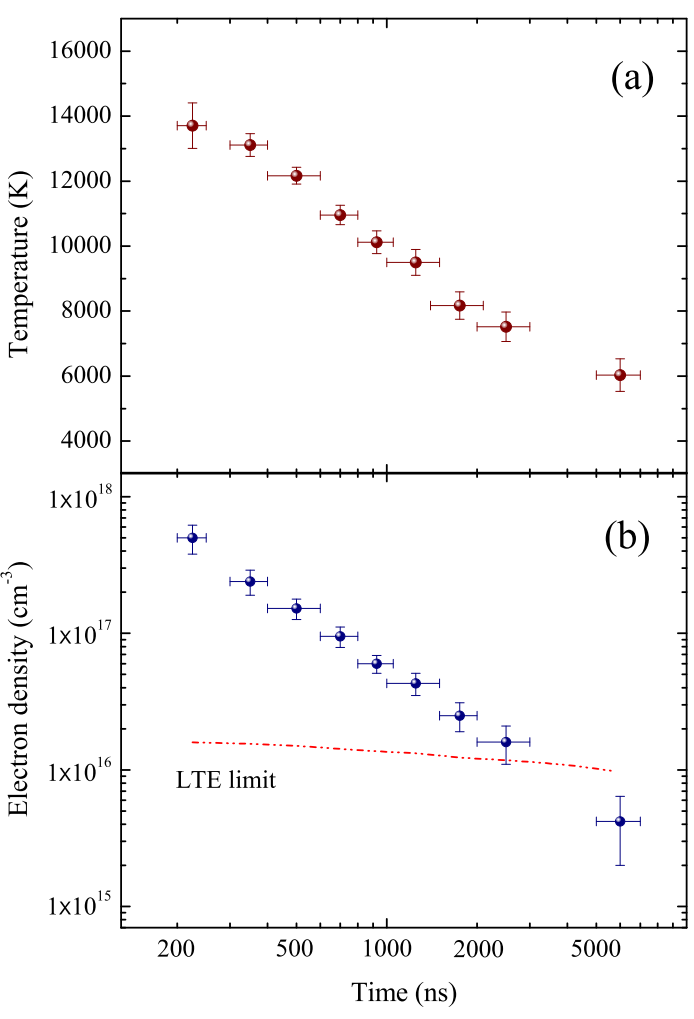

Figure 6: Temporal evolution of electron density (a) and excitation temperature (b). The dashed red line (b) stands for the minimum $n_{e^{-}}$ value required for LTE according to the McWhirter criterion [47].

dominate (see Fig. 1), the ground state population number densities of neutral atoms are small and their resonance lines have little optical thickness. Contrarily, at late times, when the temperature is low, neutral atoms dominate, their ground state population number densities are large, and self-absorption of resonance lines is strong.

For transitions of large Stark shift, the influence of the optical thickness on the spectral line shape can be verified by analyzing the correlation between Stark width and shift. This is illustrated in Fig. 7, where the linear increase of Stark shift with Stark width is observed for transitions of $\mathrm{Ca}$ and $\mathrm{Ca}^{+}$.

The spectral lines having small optical thickness over the entire time-interval were used to analyse the dependence of their Stark widths and shifts on the Stark width of the $\mathrm{H}_{\alpha}$ transition. This is shown in Fig. 8 where the Stark width of Ca I $585.74 \mathrm{~nm}$ is presented versus $\mathrm{H}_{\alpha}$ Stark width on a logarithmic scale. Assuming linear dependencies of Stark width with electron density for non-hydrogenic transitions, we can deduce from the slope the exponent $m$ that characterizes the dependence of the $\mathrm{H}_{\alpha}$ Stark width on $n_{e}$ (see Eq. 3). We observe in 


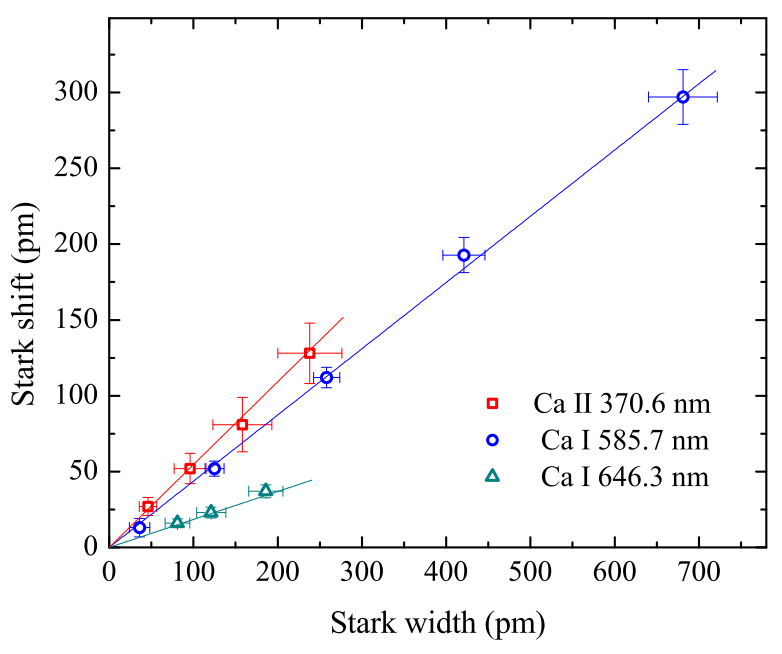

Figure 7: Stark shift vs Stark width of calcium lines deduced from measurements at various delays.
Fig. 8 two slightly different slopes, indicating that the $n_{e}$-dependence of $\mathrm{H}_{\alpha}$ Stark broadening in the low electron density range differs from that at large $n_{e}$-values. The transition between both regimes corresponds to a delay $\approx 1000 \mathrm{~ns}$ for which the electron density is close to $1 \times 10^{17} \mathrm{~cm}^{-3}$. We thus describe Stark broadening of $\mathrm{H}_{\alpha}$ using Eq. (3) with $w=1.10 \mathrm{~nm}$ according to literature [40,48] and $m=0.7$ or $m=0.9$ for electron densities below or above $n_{e}^{\text {ref }}=1 \times 10^{17} \mathrm{~cm}^{-3}$, respectively. It is noted that the expression equals that proposed by Gigosos et al. [40] and Konjevic et al. [48] for $n_{e}<1 \times 10^{17} \mathrm{~cm}^{-3}$ whereas it differs at larger electron densities by the $m$-value exclusively. According to ${ }^{399}$ the good agreement between measured and computed Stark widths for $n_{e} \approx 1 \times 10^{17} \mathrm{~cm}^{-3}$ reported in literature [10] (see section 2) we estimate the uncertainty to $\cong 10 \%$ for $n_{e}$-values close to $n_{e}^{r e f}$. The error increases with distance from the reference electron density due to the uncertainty of $m$.

\subsection{Determination of Stark broadening parameters}

After the implementation of the electron density ${ }_{409}$ measurement procedure using $\mathrm{H}_{\alpha}$, we explore now 410 the linear dependence of Stark widths and shifts on 411 $n_{e}$ for non-hydrogenic transitions to determine their ${ }_{412}$ Stark broadening parameters $w$ and $d$. Therefore, the ${ }_{413}$ Stark widths and shifts of non-hydrogenic transitions 414 were measured for the spectra recorded with different 415 delays and and plotted as functions of electron density ${ }_{416}$ as shown in Fig. 9. According to the precise linear 417 increase, the relative errors arising from the linear 418

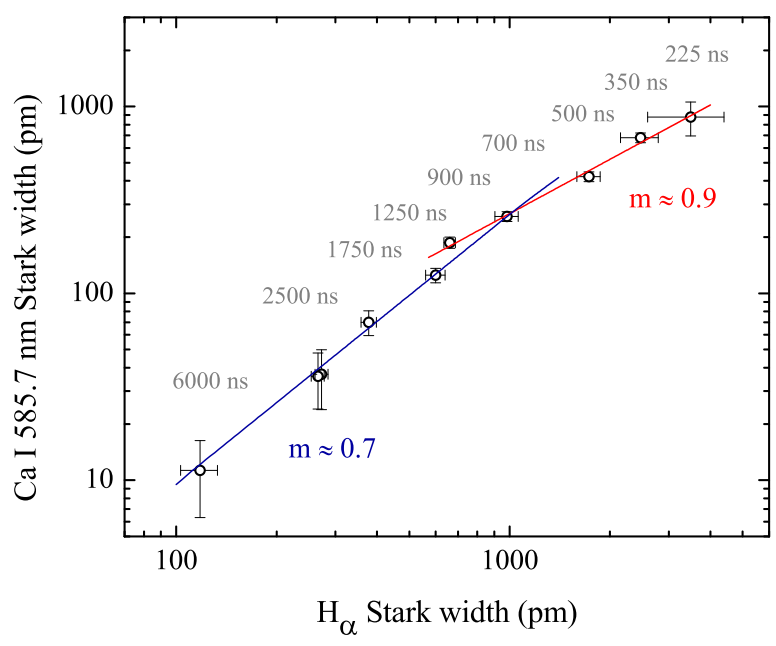

Figure 8: Stark width of a non-hydrogenic transition vs $\mathrm{H}_{\alpha}$ Stark width. We deduce slightly different $m$-values for $\mathrm{H}_{\alpha}$ Stark broadening (see Eq. 3) for the $n_{e}$-ranges below and above $1 \times 10^{17} \mathrm{~cm}^{-3}$.

analysis are small compared to the absolute errors associated to the electron density measurement using $\mathrm{H}_{\alpha}$. Thus, for isolated lines of measurable Stark width over a large $n_{e}$-range, the errors of the deduced $w$ - and $d$-values are close to those of the most accurate $n_{e^{-}}$ measurements, evaluated to about $15 \%$. For transitions having measurable Stark width in a restricted $n_{e}$-range only, the measurement errors of $w$ and $d$ are naturally larger.

The deduced broadening parameters are presented in Tables 2 and 3 for the spectral lines of calcium and other elements, respectively. Assuming an accuracy of electron density measurements of about $15 \%$ for $n_{e}$-values close to $10^{17} \mathrm{~cm}^{-3}$, the estimated $w$-measurement error ranges from 20 to $30 \%$ for most transitions. For some lines, the accuracy is lower due to larger contributions of apparatus- and/or resonance broadening to the line profile. Compared to Stark broadening parameters reported in literature (see last two columns in the Tables), a mismatch larger than the estimated accuracy is observed for several lines. Depending on the multiplet, the values reported in literature are larger or smaller than the broadening parameters we report here. The large dispersion $w$ - and $d$-values measured in different experiments is attributed to two main causes: (i) the uncertainty of electron density due to the difficulties of calibrating the $n_{e}$-measurements. Indeed, absolute values of electron density are exclusively obtained through the calculation of Stark broadening parameters leading to a large variability of $n_{e}$ that depends on the chosen 
Table 2: Wavelength $\lambda$, configuration and term of upper and lower excitation levels of transitions according to NIST [44]. The measured Stark width $w$ and shift $d$ and the values reported in literature $w^{l i t}$ and $d^{l i t}$ are given for $n_{e}=1 \times 10^{17} \mathrm{~cm}^{-3} . \Delta w / w$ and $\Delta d / d$ are the relative errors of the measured Stark width and shift, respectively.

\begin{tabular}{|c|c|c|c|c|c|c|c|c|c|c|c|}
\hline \multirow[t]{2}{*}{ Species } & \multirow{2}{*}{$\begin{array}{c}\lambda \\
(\mathrm{nm})\end{array}$} & \multicolumn{2}{|c|}{ Lower level } & \multicolumn{2}{|c|}{ Upper level } & \multirow{2}{*}{$\begin{array}{c}w \\
(\mathrm{pm})\end{array}$} & \multirow{2}{*}{$\begin{array}{c}\Delta w / w \\
(\%)\end{array}$} & \multirow{2}{*}{$\begin{array}{c}d \\
(\mathrm{pm})\end{array}$} & \multirow{2}{*}{$\begin{array}{c}\Delta d / d \\
(\%)\end{array}$} & \multirow{2}{*}{$\begin{array}{c}w^{l i t} \\
(\mathrm{pm})\end{array}$} & \multirow{2}{*}{$\begin{array}{c}d^{l i t} \\
(\mathrm{pm})\end{array}$} \\
\hline & & Config. & Term & Config. & Term & & & & & & \\
\hline \multirow[t]{5}{*}{$\mathrm{Ca} \mathrm{I}$} & 299.496 & $3 p^{6} 4 s 4 p$ & ${ }^{3} \mathrm{P}^{\mathrm{o}}$ & $3 p^{6} 3 d^{2}$ & ${ }^{3} \mathrm{P}$ & 23 & 20 & 4.4 & 30 & $29^{a}$ & - \\
\hline & 299.732 & & & & & & & & & & \\
\hline & 300.086 & & & & & & & & & & \\
\hline & 300.686 & & & & & & & & & & \\
\hline & 300.921 & & & & & & & & & & \\
\hline $\mathrm{Ca} \mathrm{I}$ & 422.673 & $3 p^{6} 4 s^{2}$ & ${ }^{1} \mathrm{~S}$ & $3 p^{6} 4 s 4 p$ & ${ }^{1} \mathrm{P}^{\mathrm{o}}$ & 32 & 60 & 6 & 40 & - & - \\
\hline \multirow[t]{4}{*}{$\mathrm{Ca} \mathrm{I}$} & 429.899 & $3 p^{6} 4 s 4 p$ & ${ }^{3} \mathrm{P}^{\mathrm{o}}$ & $3 p^{6} 4 p^{2}$ & ${ }^{3} \mathrm{P}$ & 40 & 25 & -8 & 40 & - & - \\
\hline & 430.253 & & & & & & & & & & \\
\hline & 430.774 & & & & & & & & & & \\
\hline & 431.865 & & & & & & & & & $15.5^{a}$ & - \\
\hline \multirow[t]{6}{*}{$\mathrm{Ca} \mathrm{I}$} & 442.544 & $3 p^{6} 4 s 4 p$ & ${ }^{3} \mathrm{P}^{\mathrm{o}}$ & $3 p^{6} 4 s 4 d$ & ${ }^{3} \mathrm{D}$ & 200 & 20 & - & - & - & - \\
\hline & 443.496 & & & & & & & & & & \\
\hline & 443.568 & & & & & & & & & & \\
\hline & 445.478 & & & & & & & & & & \\
\hline & 445.589 & & & & & & & & & & \\
\hline & 445.662 & & & & & & & & & & \\
\hline \multirow[t]{5}{*}{$\mathrm{Ca} \mathrm{I}$} & 558.197 & $3 p^{6} 3 d 4 s$ & ${ }^{3} \mathrm{D}$ & $3 p^{6} 3 d 4 p$ & ${ }^{3} \mathrm{D}^{0}$ & 90 & 20 & 28 & 30 & - & - \\
\hline & 558.875 & & & & & & & & & & \\
\hline & 559.011 & & & & & & & & & & \\
\hline & 559.446 & & & & & & & & & & \\
\hline & 559.848 & & & & & & & & & & \\
\hline $\mathrm{Ca} \mathrm{I}$ & 585.745 & $3 p^{6} 4 s 4 p$ & ${ }^{1} \mathrm{P}^{\mathrm{o}}$ & $3 p^{6} 4 p^{2}$ & ${ }^{1} \mathrm{D}$ & 260 & 20 & 100 & 20 & - & - \\
\hline $\mathrm{Ca} \mathrm{I}$ & 612.222 & $3 p^{6} 4 s 4 p$ & ${ }^{3} \mathrm{P}^{\mathrm{o}}$ & $3 p^{6} 4 s 5 s$ & ${ }^{3} \mathrm{~S}$ & 165 & 20 & 75 & 20 & - & - \\
\hline \multirow[t]{3}{*}{$\mathrm{Ca} \mathrm{I}$} & 643.908 & $3 p^{6} 3 d 4 s$ & ${ }^{3} \mathrm{D}$ & $3 p^{6} 3 d 4 p$ & ${ }^{3} \mathrm{~F}^{\mathrm{o}}$ & 66 & 20 & 14 & 30 & - & - \\
\hline & 646.257 & & & & & & & & & & \\
\hline & 649.378 & & & & & & & & & & \\
\hline \multirow[t]{2}{*}{ Ca II } & 210.324 & $3 p^{6} 4 p$ & ${ }^{2} \mathrm{P}^{\mathrm{o}}$ & $3 p^{6} 5 d$ & ${ }^{2} \mathrm{D}$ & 104 & 20 & 30 & 20 & $67^{b}$ & $23^{b}$ \\
\hline & 211.276 & & & & & & & & & $73^{b}$ & $26^{b}$ \\
\hline \multirow[t]{2}{*}{ Ca II } & 370.602 & $3 p^{6} 4 p$ & ${ }^{2} \mathrm{P}^{\mathrm{o}}$ & $3 p^{6} 5 s$ & ${ }^{2} \mathrm{~S}$ & 79 & 25 & 47 & 20 & $135^{d}$ & $35^{c}$ \\
\hline & 373.690 & & & & & & & & & $183^{d}$ & $35^{c}$ \\
\hline
\end{tabular}

${ }^{a}$ Ref. [25], ${ }^{b}$ Ref. [31], ${ }^{c}$ Ref. [28], ${ }^{d}$ Ref. [24]. 
Table 3: Wavelength $\lambda$, configuration and term of upper and lower excitation levels of transitions according to NIST [44]. The measured Stark width $w$ and shift $d$ and the values reported in literature $w^{\text {lit }}$ and $d^{l i t}$ are given for $n_{e}=1 \times 10^{17} \mathrm{~cm}^{-3} . \Delta w / w$ and $\Delta d / d$ are the relative errors of the measured Stark width and shift, respectively.

\begin{tabular}{|c|c|c|c|c|c|c|c|c|c|c|c|}
\hline \multirow[t]{2}{*}{ Species } & \multirow{2}{*}{$\begin{array}{c}\lambda \\
(\mathrm{nm})\end{array}$} & \multicolumn{2}{|c|}{ Lower level } & \multicolumn{2}{|c|}{ Upper level } & \multirow{2}{*}{$\begin{array}{c}w \\
(\mathrm{pm})\end{array}$} & \multirow{2}{*}{$\begin{array}{c}\Delta w / w \\
(\%)\end{array}$} & \multirow{2}{*}{$\begin{array}{c}d \\
(\mathrm{pm})\end{array}$} & \multirow{2}{*}{$\begin{array}{c}\Delta d / d \\
(\%)\end{array}$} & \multirow{2}{*}{$\begin{array}{c}w^{\text {lit }} \\
(\mathrm{pm})\end{array}$} & \multirow{2}{*}{$\begin{array}{c}d^{l i t} \\
(\mathrm{pm})\end{array}$} \\
\hline & & Config. & Term & Config. & Term & & & & & & \\
\hline C I & 247.856 & $2 s^{2} 2 p^{2}$ & ${ }^{1} \mathrm{~S}$ & $3 s^{2} 2 p 3 s$ & ${ }^{3} \mathrm{P}^{\mathrm{o}}$ & 14 & 30 & 8 & 25 & $6.8^{e}$ & $2.6^{e}$ \\
\hline $\mathrm{Cu}$ II & $\begin{array}{l}212.604 \\
213.598\end{array}$ & $3 d^{9}\left({ }^{2} \mathrm{D}\right) 4 s$ & ${ }^{3} \mathrm{D}$ & $3 d^{9}\left({ }^{2} \mathrm{D}\right) 4 p$ & ${ }^{3} \mathrm{~F}^{\mathrm{o}}$ & 6 & 30 & 1.1 & 50 & $8.2^{f}$ & - \\
\hline Mg I & $\begin{array}{l}277.669 \\
277.827 \\
278.141 \\
278.297\end{array}$ & $3 s 3 p$ & ${ }^{3} \mathrm{P}^{\mathrm{o}}$ & $3 p^{2}$ & ${ }^{3} \mathrm{P}$ & 7 & 40 & 1 & 100 & - & - \\
\hline Mg I & 285.212 & $2 p^{6} 3 s^{2}$ & ${ }^{1} \mathrm{~S}$ & $3 s 3 p$ & ${ }^{1} \mathrm{P}^{\mathrm{o}}$ & 17 & 30 & 8.5 & 30 & - & - \\
\hline $\mathrm{Mg} \mathrm{I}$ & $\begin{array}{l}382.935 \\
383.230 \\
383.829\end{array}$ & $3 s 3 p$ & ${ }^{3} \mathrm{P}^{\mathrm{o}}$ & $3 s 3 d$ & ${ }^{3} \mathrm{D}$ & 270 & 20 & -45 & 30 & $110^{g}$ & $-2^{g}$ \\
\hline $\mathrm{Mg} \mathrm{I}$ & $\begin{array}{l}516.732 \\
517.268 \\
518.360\end{array}$ & $3 s 3 p$ & ${ }^{1} \mathrm{P}^{\mathrm{o}}$ & $3 s 4 s$ & ${ }^{3} \mathrm{~S}$ & 90 & 20 & 50 & 20 & $\begin{array}{r}33^{g} \\
35.5^{g} \\
35^{g}\end{array}$ & $\begin{array}{r}9^{g} \\
8.4^{g} \\
7.4^{g}\end{array}$ \\
\hline $\mathrm{Mg}$ II & $\begin{array}{l}279.077 \\
279.799\end{array}$ & $2 p^{6} 3 p$ & ${ }^{2} \mathrm{P}^{\mathrm{o}}$ & $2 p^{6} 3 d$ & ${ }^{2} \mathrm{D}$ & 30 & 20 & 9 & 25 & $\begin{array}{l}162^{h} \\
144^{h}\end{array}$ & $\begin{array}{l}22^{h} \\
19^{h}\end{array}$ \\
\hline Mg II & $\begin{array}{l}292.863 \\
293.651\end{array}$ & $2 p^{6} 3 p$ & ${ }^{2} \mathrm{P}^{\mathrm{o}}$ & $2 p^{6} 4 s$ & ${ }^{2} \mathrm{~S}$ & 50 & 20 & 23 & 20 & $\begin{array}{l}29^{i} \\
30^{i}\end{array}$ & $\begin{array}{l}57^{i} \\
68^{i}\end{array}$ \\
\hline Si I & $\begin{array}{l}250.689 \\
251.431 \\
251.611 \\
251.920 \\
252.410 \\
252.850\end{array}$ & $3 s^{2} 3 p^{2}$ & ${ }^{3} \mathrm{P}$ & $3 s^{2} 3 p 4 s$ & ${ }^{3} \mathrm{P}^{\mathrm{o}}$ & 14 & 25 & 8 & 25 & $\begin{array}{l}14.1^{j} \\
11.2^{j} \\
11.7^{j} \\
11.2^{j} \\
10.4^{j} \\
10.7^{j}\end{array}$ & - \\
\hline $\mathrm{Fe}$ II & $\begin{array}{l}238.203 \\
238.862 \\
239.562 \\
239.924 \\
240.488 \\
241.051\end{array}$ & $3 d^{6}\left({ }^{5} \mathrm{D}\right) 4 s$ & $a^{6} D$ & $3 d^{6}\left({ }^{5} \mathrm{D}\right) 4 p$ & $z^{6} F^{o}$ & 5 & 30 & 2 & 30 & - & - \\
\hline $\mathrm{Fe}$ II & $\begin{array}{l}259.587 \\
259.836 \\
259.939 \\
260.708 \\
261.187 \\
261.382\end{array}$ & $3 d^{6}\left({ }^{5} \mathrm{D}\right) 4 s$ & $a^{6} D$ & $3 d^{6}\left({ }^{5} \mathrm{D}\right) 4 p$ & $z^{6} D^{o}$ & 6 & 30 & 0.8 & 60 & $\begin{array}{r}- \\
4.5^{k}\end{array}$ & - \\
\hline Fe II & 273.954 & $3 d^{6}\left({ }^{5} \mathrm{D}\right) 4 s$ & $a^{4} D$ & $3 d^{6}\left({ }^{5} \mathrm{D}\right) 4 p$ & $z^{4} D^{o}$ & 9 & 30 & 3 & 35 & $5.3^{l}$ & - \\
\hline O I & $\begin{array}{l}777.194 \\
777.416 \\
777.538\end{array}$ & $2 s^{2} 2 p^{3}\left({ }^{4} S^{o}\right) 3 s$ & ${ }^{5} S^{0}$ & $2 s^{2} 2 p^{3}\left({ }^{4} S^{o}\right) 3 p$ & ${ }^{5} \mathrm{P}$ & 105 & 35 & 15 & 40 & - & - \\
\hline Sr II & $\begin{array}{l}407.770 \\
421.551\end{array}$ & $4 p^{6} 5 s$ & ${ }^{2} \mathrm{~S}$ & $4 p^{6} 5 p$ & ${ }^{2} \mathrm{P}^{\mathrm{o}}$ & 41 & 20 & -3.4 & 30 & - & - \\
\hline
\end{tabular}

${ }^{e}$ Ref. [49], ${ }^{f}$ Ref. [50], ${ }^{g}$ Ref. [51], ${ }^{h}$ Ref. [52], ${ }^{i}$ Ref. [53], ${ }^{j}$ Ref. [54], ${ }^{k}$ Ref. [55], ${ }^{l}$ Ref. [56]. 


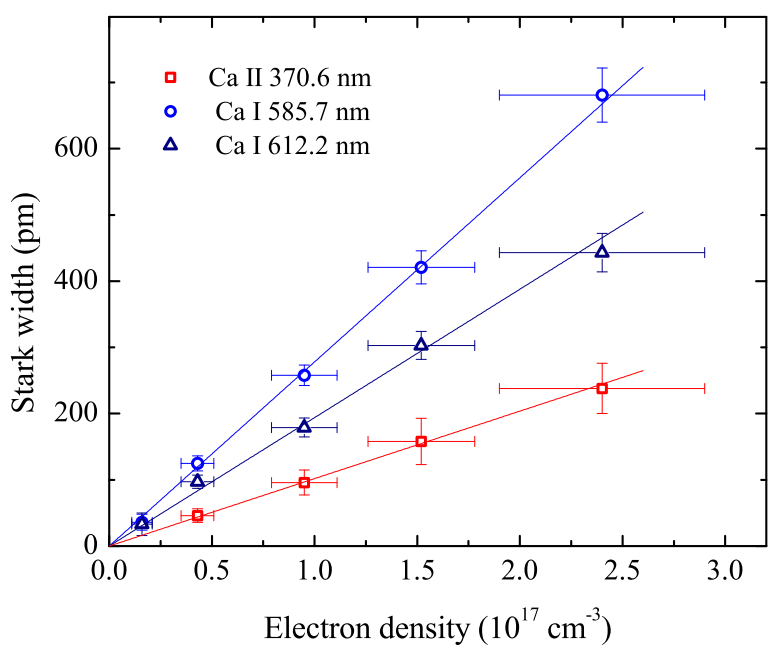

Figure 9: Stark widths of Ca I and Ca II transitions vs electron density. 466

transition and the $n_{e}$-range. (ii) The measurements reported in literature were performed using various types ${ }^{468}$ of plasmas characterized by different temperatures. Although the $T$-dependence was neglected in the present ${ }_{469}$ work according to the moderate temperature variation in 470 the laser-produced plasma, the changes of Stark broad- 471 ening parameters with temperature cannot be neglected 472 in case of strong $T$-variation.

As an example, the large variability of Stark broadening 474 parameters in literature is illustrated by the $w$-values of 475 6.8 and $54 \mathrm{pm}$ reported for C I $247.85 \mathrm{~nm}[3,49]$. We ${ }_{476}$ stress that the most intense spectral lines (see Fig. 2) 477 were not considered as their large optical thickness pre- ${ }^{478}$ vent the accurate Stark width measurement.

\section{Conclusion}

We presented a method for the measurement of Stark broadening parameters based on modeling of the emis- ${ }^{486}$ sion spectrum from a laser-induced plasma. Produc- ${ }^{487}$ ing ablation with ultraviolet nanosecond laser pulses in ${ }_{489}^{488}$ argon at near atmospheric pressure, the measurements 490 take advantage of the spatially uniform distributions of 491 electron density and temperature within the ablated va- ${ }_{493}^{492}$ por plume. These properties enable simple and accurate ${ }_{494}^{493}$ modeling based on the calculation of the spectral radi- 495 ance of a plasma in local thermodynamic equilibrium. ${ }^{496}$ The spectra recording with an echelle spectrometer of ${ }_{498}^{497}$ large resolving power give access to analysis of a large ${ }_{499}$ number of spectral lines. Using hydrated calcium sul- 500 phate as sample material, we were able to deduce the ${ }^{501}$ Stark broadening parameters of atomic and ionic spec- ${ }_{503}^{502}$ tral lines from calcium, oxygen and several impurity el- 504 ements by their simultaneous observation with the $\mathrm{H}_{\alpha}$ transition. By varying the delay of the detector gate with respect to the laser pulse, the electron density was varied by more than two orders of magnitude from $3 \times 10^{15}$ to $5 \times 10^{17} \mathrm{~cm}^{-3}$ whereas temperature was changed from about 6,000 to $14,000 \mathrm{~K}$. Assuming a linear increase of Stark widths of non-hydrogenic lines with $n_{e}$, the present analysis indicate a change of the $\mathrm{H}_{\alpha}$ Stark width-dependence on $n_{e}$ that occurs when the electron density varies from values $<10^{17} \mathrm{~cm}^{-3}$ to larger density. For $n_{e}>10^{17} \mathrm{~cm}^{-3}$, we observe a more non-hydrogenic behaviour whereas the typical $n_{e}^{2 / 3}$-dependence was retrieved for the low electron density range, in agreement with theoretical predictions. According to the precise electron density measurements for $n_{e}$-values close to $1 \times 10^{17} \mathrm{~cm}^{-3}$, the deduced Stark broadening parameters have fair uncertainties of 20 to $30 \%$ for most of the investigated transitions.

\section{Acknowledgements}

The authors acknowledge the financial support provided by LASERLAB- EUROPE (grant agreement No. 284464, project CNRS-LP3002148). This research has also been supported by the Ministry of education, science and technological development of the Republic of Serbia (project ON171008).

[1] H. R. Griem, Plasma spectroscopy, Academic Press, New York, 1964.

[2] H. R. Griem, Spectral line broadening of plasmas, Academic Press, New York, 1974.

[3] N. Konjević, J. R. Roberts, A critical review of the Stark widths and shifts of spectral lines from non-hydrogenic atoms, J. Phys. Chem. Ref. Data 5 (1976) 209-257.

[4] N. Konjević, M. S. Dimitrijević, W. L. Wiese, Experimental Stark widths and shifts for spectral lines of neutral atoms (A critical review of selected data for the period 1976 to 1982), J. Phys. Chem. Ref. Data 13 (1984) 619-647.

[5] N. Konjević, A. Lesage, J. R. Führ, W. L. Wiese, Experimental Stark widths and shifts for spectral lines of neutral and ionized atoms (A critical review of selected data for the period 1983 through 1988), J. Phys. Chem. Ref. Data 19 (1990) 1307-1385.

[6] N. Konjević, A. Lesage, J. R. Führ, W. L. Wiese, Experimental Stark widths and shifts for spectral lines of neutral and ionized atoms (A critical review of selected data for the period 1989 through 2000), J. Phys. Chem. Ref. Data 31 (2002) 819-921.

[7] A. Lesage, Experimental Stark widths and shifts for spectral lines of neutral and ionized atoms A critical review of selected data for the period 2001-2007, N. Astron. Rev. 52 (2009) 471535 .

[8] A. Mendys, K. Dzierżęga, M. Grabiec, S. Pellerin, B. P. G., Travaillé, B. Bousquet, Investigations of laser-induced plasma in argon by thomson scattering, Spectrochim. Acta Part B: Atom. Spectrosc. 66 (2011) 691-697.

[9] M. Cvejić, K. Dzierżęga, T. Pięta, Investigation of thermodynamic equilibrium in laser-induced aluminum plasma using the 
$\mathrm{H}_{\alpha}$ line profiles and Thomson scattering spectra, Appl. Phys. 570 Lett. 107 (2015) 024102 1-4.

[10] H. Griem, Stark broadening of the hydrogen Balmer- $\alpha$ line in 572 low and high density plasmas, Contrib. Plasma Phys. 40 (2000) 573 46-56.

[11] S. Eliezer, A. D. Krumbein, D. Salzmann, Generalized valid- 575 ity condition for local thermodynamic-equilibrium in a laser- 576 produced plasma, J. Phys. D: Appl. Phys. 11 (1978) 1693-1701. 577

[12] G. Cristoforetti, E. Tognoni, L. A. Gizzi, Thermodynamic equi- 578 librium states in laser-induced plasmas: From the general case to 579 laser-induced breakdown spectroscopy plasmas, Spectrochim. 580 Acta Part B: Atom. Spectrosc. 90 (2013) 1-22.

[13] M. Boueri, M. Baudelet, J. Yu, X. Mao, S. S. Mao, R. Russo, 582 Early stage expansion and time-resolved spectral emission of 583 laser-induced plasma from polymer, Appl. Surf. Sci. 255 (2009) 584 9566-9571.

[14] Q. Ma, V. Motto-Ros, F. Laye, J. Yu, W. Lei, X. Bai, L. Zheng, 586 H. Zeng, Ultraviolet versus infrared: Effects of ablation laser 587 wavelength on the expansion of laser-induced plasma into one- 588 atmosphere argon gas, J. Appl. Phys. 111 (2012) 1-11. 589

[15] I. N. Mihailescu, J. Hermann, Laser-plasma interactions, in: 590 P. Schaaf (Ed.), Laser Processing of Materials, Springer, Berlin, 591 2010, pp. 49-88.

[16] M. Burger, D. Pantić, Z. Nikolić, S. Djeniže, Shielding effects 593 in the laser-generated copper plasma under reduced pressures of 594 He atmosphere, J. Quant. Spectrosc. Radiat. Transf. 170 (2016) 595 19-27.

[17] L. Mercadier, J. Hermann, C. Grisolia, A. Semerok, Diagnostics 597 of nonuniform plasmas for elemental analysis via laser-induced 598 breakdown spectroscopy: demonstration on carbon-based mate- 599 rials, J. Anal. At. Spectrom. 28 (2013) 1446-1455.

[18] J. Hermann, C. Gerhard, E. Axente, C. Dutouquet, Comparative 601 investigation of laser ablation plumes in air and argon by anal- 602 ysis of spectral line shapes: Insights on calibration-free laser- 603 induced breakdown spectroscopy, Spectrochim. Acta Part B: 604 Atom. Spectrosc. 100 (2014) 189-196.

[19] M. S. Dimitrijević, S. Sahal-Bréchot, Stark broadening of neu- 606 tral calcium spectral lines, Astron. Astrophys. Supp. 140 (1999) 607 191-192.

[20] J. Chapelle, S. Sahal-Bréchot, Experimental and theoretical 609 electron impact broadening of some $\mathrm{Mg}$ II and Ca II lines of 610 astrophysical interest, Astron. Astrophys. 6 (1970) 415-422.

[21] J. S. Hildum, J. Cooper, Stark broadening of calcium ion reso- 612 nance lines, Phys. Lett. 36A (1971) 153-155.

[22] J. Purić, N. Konjević, Stark shifts of some isolated spectral lines 614 of singly ionized earth alkaline metals, Z. Phys. 249 (1972) 440_ 615 444.

[23] W. W. Jones, A. Sanchez, J. R. Greig, H. R. Griem, Measure- 617 ment and calculation of the Stark-broadening parameters for the 618 resonance lines of singly ionized calcium and magnesium, Phys. 619 Rev. A 5 (1972) 2318-2328.

[24] H. Kusch, H. Pritschow, Broadening and shift of calcium lines by microfields, Astron. Astrophys. 4 (1970) 31-35.

25] R. Hühn, H. Kusch, Broadening and shift of calcium lines by 622 van der Waals interaction with argon atoms and by electron im- 624 pact, Astron. Astrophys. 28 (1973) 159-164.

[26] D. Hadžiomerspahić, M. Platiša, N. Konjević, M. Popović, Stark 626 broadening and shift of some isolated spectral lines of singly 627 ionised earth alkaline metals, Z. Phys. 262 (1973) 169-179.

[27] J. F. Baur, J. Cooper, A shock tube study of line broadening 629 in a temperature range of 6100 to $8300 \mathrm{~K}$, J. Quant. Spectrosc. 630 Radiat. Transf. 17 (1977) 311-322.

[28] C. Fleurier, S. Sahal-Bréchot, J. Chapelle, Stark profiles of some 632 ion lines of alkaline earth elements, J. Quant. Spectrosc. Radiat. 633 Transf. 17 (1977) 595-604.
[29] C. Goldbach, G. Nollez, P. Plomdeur, J.-P. Zimmermann, Starkwidth measurements of singly ionized calcium resonance lines in a wall-stabilized arc, Phys. Rev. A 28 (1983) 234-237.

[30] A. Srećković, S. Djeniže, Measured Stark width and shift of $393.367 \mathrm{~nm}$ Ca II resonance spectral line, Bull. Astron. Belgr. 148 (1993) 7-10.

[31] J. A. Aguilera, C. Aragón, J. Manrique, Measurement of Stark widths and shifts of Ca II spectral lines, MNRAS 444 (2014) 1854-1858.

[32] M. S. Dimitrijević, S. Sahal-Bréchot, Stark broadening parameter tables for neutral calcium spectral lines, Serb. Astron. J. 161 (2000) 39-88.

[33] M. S. Dimitrijević, S. Sahal-Bréchot, Stark broadening parameter tables for $\mathrm{Ca}$ II lines of astrophysical interest, Bull. Astron. Belgr. 145 (1992) 81-99.

[34] M. S. Dimitrijević, S. Sahal-Bréchot, Stark broadening of $\mathrm{Ca}$ II spectral lines, J. Quant. Spectrosc. Radiat. Transf. 49 (1993) $157-164$.

[35] I. Tapalaga, I. P. Dojčinović, M. K. Milosavljević, J. Purić, Stark width regularities within neutral calcium spectral series, Publ. Astron. Soc. Aust. 29 (2012) 20-28.

[36] M. Cirisan, M. Cvejić, M. Gavrilović, S. Jovicević, N. Konjević, J. Hermann, Stark broadening measurement of Al II lines in a laser-induced plasma, J. Quant. Spectrosc. Radiat. Transf. 133 (2014) 652-662.

[37] C. Gerhard, J. Hermann, L. Mercadier, L. Loewenthal, E. Axente, C. Luculescu, T. Sarnet, M. Sentis, W. Viöl, Quantitative analyses of glass via laser-induced breakdown spectroscopy in argon, Spectrochim. Acta Part B: Atom. Spectrosc. 101 (2014) $32-45$.

[38] X. Z. Zhao, L. J. Shen, T. X. Lu, K. Niemax, Spatial distributions of electron-density in microplasmas produced by laser ablation of solids, Appl. Phys. B: Photophys. Laser Chem. 55 (1992) 327-330

[39] E. Tognoni, V. Palleschi, M. Corsi, G. Cristoforetti, N. Omenetto, I. Gornushkin, B. W. Smith, J. D. Winefordner, From sample to signal in laser-induced breakdown spectroscopy: a complex route to quantitative analysis, in: A. W. Miziolek, V. Palleschi, I. Schechter (Eds.), Laser-induced breakdown spectroscopy, Cambridge University, Berlin, 2006, pp. 122-194.

[40] M. A. Gigosos, M. A. Gonzalez, V. Cardenoso, Computer simulated Balmer-alpha, -beta, and -gamma Stark line profiles for non-equilibrium plasma diagnostics, Spectrochim. Acta Part B: Atom. Spectrosc. 58 (2003) 1489-1504.

[41] A. D. Giacomo, M. Dell'Aglio, R. Gaudiuso, G. Cristoforetti, S. Legnaioli, V. Palleschi, E. Tognoni, Spatial distribution of hydrogen and other emitters in aluminum laser-induced plasma in air and consequences on spatially integrated laser-induced breakdown spectroscopy measurements, Spectrochim. Acta Part B: Atom. Spectrosc. 63 (9) (2008) 980-987.

[42] J. Hermann, A. Lorusso, A. Perrone, F. Strafella, C. Dutouquet, B. Torralba, Simulation of emission spectra from nonuniform reactive laser-induced plasmas, Phys. Rev. E 92 (2015) 1-15.

[43] A. Radzig, B. Smirnov, Reference Data on Atoms, Molecues and Ions, Springer, Berlin, 1985.

[44] A. Kramida, Y. Ralchenko, J. Reader, Nist Atomic Spectra Database, National Institute of Standards and Technology, Gaithersburg, MD (2016).

URL http://physics.nist.gov/asd

[45] M. Burger, M. Skočić, M. Ljubisavljević, Z. Nikolić, S. Djeniže, Spectroscopic study of the laser-induced indium plasma, Eur. Phys. J. D 68 (2014) 223:1-8.

[46] D. Bulajic, M. Corsi, G. Cristoforetti, S. Legnaioli, V. Palleschi, A. Salvetti, E. Tognoni, A procedure for correcting self- 
absorption in calibration free-laser induced breakdown spectroscopy, Spectrochim. Acta Part B: Atom. Spectrosc. 57 (2002) 339-353.

[47] R. McWhirter, Ch. 5, in: R. H. Huddlestone (Ed.), Plasma Diagnostic Techniques, Academic, New York, 1965, pp. 201 - 264.

[48] N. Konjević, M. Ivković, N. Sakan, Hydrogen Balmer lines for low electron density plasma diagnostics, Spectrochim. Acta Part B: Atom. Spectrosc. 76 (2012) 16-26.

[49] S. Djeniže, A. Srećković, S. Bukvić, The C I $247.8561 \mathrm{~nm}$ resonance line stark broadening parameters, Z. Naturfors. Sect. A-J Phys. Sci. 61 (2006) 91-94.

[50] M. Skočić, M. Burger, Z. Nikolić, S. Bukvić, S. Djeniže, Stark broadening in the laser-induced $\mathrm{Cu}$ I and $\mathrm{Cu}$ II spectra, J. Phys. B 46 (2013) 185701:1-6.

[51] S. Djeniže, S. Bukvić, A. Srećković, Stark broadening and transition probability ratios in the $\mathrm{Mg}$ I spectrum, Astron. Astrophys. 425 (2004) 361-365.

[52] S. Bukvić, A. Srećković, S. Djeniže, Mg II h and k lines Stark parameters, New Astron. 9 (2004) 629-633.

[53] S. Djeniže, S. Bukvić, A. Srećković, M. Platiša, Mg II spectral line broadening in helium, oxygen and argon-helium plasmas, Astron. Astrophys. 424 (2004) 561-564.

[54] S. Bukvić, S. Djeniže, A. Srećković, Line broadening in the Si I, Si II, Si III, and Si IV spectra in the helium plasma, Astron. Astrophys. 508 (2009) 491-500.

55] C. Aragón, J. Aguilera, J. Manrique, Measurement of Stark broadening parameters of Fe II and Ni II spectral lines by laser induced breakdown spectroscopy using fused glass samples, J. Quant. Spectrosc. Radiat. Transf. 134 (2014) 39-45.

[56] C. Aragón, P. Vega, J. Aguilera, Stark width measurements of Fe II lines with wavelengths in the range 260-300 nm, J. Phys. B 44 (2011) 55002:1-7. 